As gold alloys containing too little silver cannot be parted by means of nitric acid, so the separation of lead oleate from lead stearate fails if too little of the former is present. I published, two years ago, a short article in the Chem. News, 66, 259, dealing with this subject, and refer to the same.

\title{
ACETIC ACID IN VINEGAR.
}

BY ALBERT R. LEEDS.

Received May 21, , 1895 .

R ECENTLY, having occasion to determine the acetic acid in $R$ many samples of vinegar, I was surprised to find how vague and unsatisfactory are the directions relating to this simple matter in many text-books and manuals for public analysts.

One of the latter recommends that fifty $\mathrm{cc}$. of vinegar should be used and the titration performed with soda, no particular strength or indicator being mentioned. Another, in general terms, calls attention to the fact that only approximate results can be obtained with soda, its acetate having a feeble alkaline reaction. The indicator, in the presence of which it so reacts, is not stated. Sutton's Volumetric Analysis calls attention to the necessity of substituting phenolphthalein for litmus, the acetates of the alkalies and alkaline earths having a perfectly neutral reaction to the former. He further adds that colored vinegars may be so titrated even when highly diluted.

Beginning with twenty-five cc., which $I$ found at once to be much too large a quantity, and using seminormal acid soda as a convenient strength of alkali, I presently reduced the volume of vinegar to ten $c c$. and finally to five cc. When this was diluted with fifty cc. of water in a white porcelain dish, and phenolphthalein was used as indicator, the end reaction was satisfactory, except in the case of highly colored vinegars. In these it is not sharp. Nor can litmus paper or litmus solution be satisfactorily used with them. Litmus gives not only a lower reading, but lower by a very variable amount in different vinegars. Sixteen samples tested as above described gave percentages ranging from 2.63 per cent. to 5.65 per cent., with a mean of 4.49 . A mixture made up of twenty-five cc. of each, gave 4.4 .4 per cent. The color of these samples was white to dark brown. 
According to Sutton, Pettenkoffer titrates vinegar with a known excess of baryta and estimates the excess with decinormal nitric or oxalic acid by the help of turmeric paper.

Thinking that baryta might give a sharper reaction, though not discerning any advantage in the use of decinormal oxalic or nitric acid, I used an approximately decinormal baryta solution with phenolphthalein and decinormal hydrochloric acid. The results agreed to o.o per cent. with those obtained with soda, and ordinarily the latter would be the more convenient and preferable reagent.

Sutton recommends as a process applicable in all cases and however dark the vinegar may be, the method of $\mathrm{C}$. Mohr, which consists in adding to a known quantity of the vinegar a known excess of barium or calcium carbonate in fine powder. Pure calcium carbonate, he states, is preferable, as it dissolves more readily.

In his excellent manual on "Foods" page 5 I 8 , Blyth adds: "If absolutely accurate determinations are required, it is best to add an excess of pure calcium carbonate to a known weight; the liquid is boiled, filtered, and the residual carbonate dissolved in slight excess of normal hydrochloric acicl and titrated back with soda and cochineal. From the amotnet of carbonate thus found to have been unacted upon, the total acidity is calculated."

This process failed entirely in my hands; I took fifty ce. of the vinegar and added two and a half grams of pure precipitated calcium carbonate in fine powder. The flask was corked loosely and shaken many times each day during the course of six days, at the end of which time the contents were still acid. Also, the flask was grently heated at intervals some five to six hours in all. The acetic acid still remaining, after filtering off the calcium carbonate, amounted to nine-tenths per cent. The figure calculated from the residual carbonate corresponded to 3.64 per cent., or a total of 4.54 per cent. as agrainst $4.4+$ per cent., tound directly with soda.

Regarding the amount of vinegar as too large and the difficulty as probably due to the slowness with which a very dilute solution of acid decomposed the carbonate towards the end of the reaction, I then treated ten $c c$. with excess of carbonate for 
two hours under a return-condenser. This was somewhat more successful, the liquid containing 0.72 per cent. and the figure calculated from the residual carbonate corresponded to 3.85 per cent.-a total of 4.57 per cent.

The true percentage being thus rendered very doubtful, I then had recourse to another recommended by Blyth. He states, page $5 \mathrm{I} 7$, that the strength may be accurately estimated by distilling IIO cc. until I0O cc. have been drawn over, i. e., ten-elevenths." The $100 \mathrm{cc}$. will contain eighty per cent. of the entire acid present in the I Io cc., and may be titrated; or the specific gravity of the distillate may be taken and the strength found from the following table: "A trial by this method yielded a distillate with specific gravity of 1.055 at $15^{\circ}$, corresponding in the table given to 4.25 per cent. ; in one trial, the distillate should by titration, 4.23 per cent., in another, 4.24. Assuming for reasons stated below, that the correct percentage was 4.43 , these figures correspond to ninety-six per cent. of the acid instead of eighty.

The method of distillation was then varied in the following manner: Ten cc. were diluted with fifty cc. of water in a small retort the neck of which was inclined upward and then bent downward to join the condenser. The distillation was continued until about two cc. remained in the retort when another fifty cc. of water was added and the distillation repeated-in all four times. The distillates had a faint acetous, mingled with a pronounced hop-like odor, on adding silver nitrate they presently darkened and on standing threw down a black deposit. They contained :

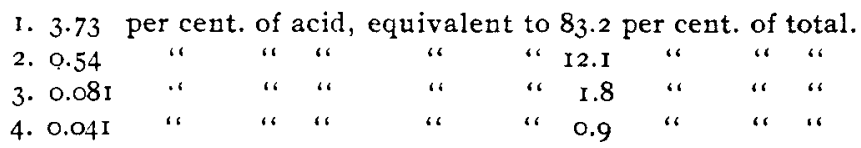

During this long process I had obtained only 4.39 per cent. acid or ninety-nine per cent. of the total.

The distillation was repeated in the same manner, using ten -cc. of vinegar and fifty cc. of water in three successive additions, but the vinegar had previously been strongly fortified with phosphoric acid. One trial gave 4.507 per cent. acid, another $4.5^{\mathrm{I}} 4$. 
The distillates gave no reaction for chlorine, and possibly the high figure was due to the presence of a little acetate.

Finally, in order to get rid of the disturbing influence of the coloring mattersf the following method was adopted. Fifty cc. of vinegar with fifty cc., of water and a drop of phenolphthalein were titrated with decinormal baryta, the latter being added to three cc. in excess, and then followed by five cc. of decinormal sulphuric acid. Whether the precipitates were allowed to settie in the cold or with warming, appeared to make no difference in the results, the coloring-matters being carried down very nicely. In one trial the precipitate was filtered off and thoroughly washed before titrating back with acid: in another, the assay was made up to $100 \mathrm{cc}$. in a graduate and twenty-five cc. wete pipetted off and titrated. The former corresponded to 4.48 per cent., the latter to 4.52 per cent. The bulk of the precipitate was approximately one cc., and making the requisite correction gives us 4.48 for the latter result also.

These figures being higher than those obtained directly; I then went back to the use of turmeric, so highly recommended by Pettenkofer, and obtained 4.43 per cent. With great care in the observation of the first brown ring which forms in the presence of the slightest excess of baryta, when a drop of the titrated solution is brought upon turmuric paper, the end reaction can be detected with certainty. The reading is lower than that with phenoiphthalein and may be relied upon even with highly colored vinegars. For ordinary purposes, however, the latter indicator with decinormal soda is adequate.

\section{NEW BOOKS.}

An Elementary Course in Experimgntal and Analytical ChemisTRY. BY JOHN H. LONG, M.S., SC.D., PROFF,SSOR OF CHEMISTRY AND DIRECTOR OF THE CHEMICAL LABORATORJES OF THE SCHOOJS OF MHDICINE AND PHARMACY OF NORTHWESTER UNIVERSITY. pp. 507. Chicago: E. H. Colegrove \& Co.

This book considers three subjects. Part I, covering 275 pages, is devoted to experimental chemistry. The first chapter presents experiments in crystallization, in distillation, and those 